\title{
Analysis of a Braking System on the Basis of Structured Analysis Methods
}

\author{
Ben Salem J. \\ Research Unit Signals and \\ Mechatronic Systems, SMS, \\ UR13ES49 \\ National Engineering School of \\ Carthage, ENICarthage \\ University of Carthage
}

\author{
Lakhoua M.N. \\ Research Unit Signals and \\ Mechatronic Systems, SMS, \\ UR13ES49 \\ National Engineering School of \\ Carthage, ENICarthage \\ University of Carthage
}

\author{
El Amraoui L. \\ Research Unit Signals and \\ Mechatronic Systems, SMS, \\ UR13ES49 \\ National Engineering School of \\ Carthage, ENICarthage \\ University of Carthage
}

\begin{abstract}
In this paper, we present the general context of the research in the domain of analysis and modeling of mechatronic systems. In fact, we present à bibliographic review on some works of research about the systemic analysis of mechatronic systems. To better understand its characteristics, we start with an introduction about mechatronic systems and various fields related to these systems, after we present a few analysis and design methods applied to mechatronic systems. Finally, we apply the two methods SADT (Structured Analysis Design Technique) and SA-RT (Structured Analysis Real Time) to the Anti-lock Braking System (ABS).
\end{abstract}

Keywords-mechatronic system; ABS braking system; analysis and modeling; SADT method; SA-RT method

\section{INTRODUCTION}

The systemic approach is used to define a system of analyzing it as a whole and in its sub-systems of studying and measure their goals or teleology to analyze the elements of the system that supports the achievement its objectives, analyze the links, interactions, mechanisms, and factors of balance and imbalance that regulate the system's objectives with its environment or with other systems with which it interacts [1].

The systemic analysis is defined as a discipline that studied and analyzes the concept of the systems with the aim of solving complex problems, to treat jointly effects and causes. So it's a discipline that brings together theoretical, practical and methodological approaches, for studying complex systems [2].

There are several methods of analysis and design systemic allowing the modeling of a system and its technical specification.

The purpose of this paper is to present the interests of the systemic approach, based on the two methods SADT (Structured Analysis Design Technique) and SA-RT (Structured Analysis Real Time). A case study of an ABS braking system is presented and discussed.

\section{ANALYSIS AND DESIGN OF MECHATRONIC SYSTEMS}

The Mechatronics term was introduced by an engineer of the Japanese company Yaskawa Electric Corporation in 1969 for characterizing a system integrating a mechanical part and an electronic part. Recently, the definition of mechatronics has evolved with the increasing complexity of systems [3]. Indeed, several definitions exist in the literature:

The definition of the newspaper IEEE Transactions on mechatronics or that chosen in 2000 by the Technical Committee on Mechatronics Systems define the mechatronic as the integration of mechanical and electromechanical (mechanical components, machinery...) with electronic systems (microelectronics, power electronics sensors, actuators) and information technology (Systems theory, modeling, automation, software, artificial intelligence) [3].

Mechatronic systems have emerged to meet the new requirements in terms of performance, comfort, safety and energy (Fig. 1). The mechatronics brings undeniable benefits such as: reduced costs, customer satisfaction, by the proposed innovative solutions, the positive response to social requirements increasingly important: pollution, consumption, safety of passengers...

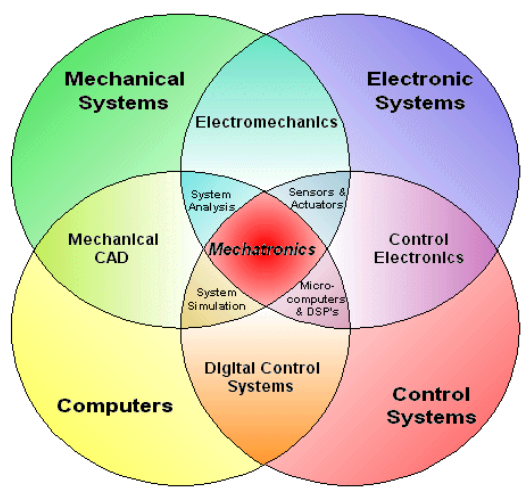

Fig. 1. Structure of a mechatronic system [4]

Nowadays, mechatronics is very present in the daily lives, as well as in industry. It touches many applications in various fields; it affects more and more the world of transport and particularly the automotive sector [5].

In this paragraph, a literature research work interested by the application of SADT and SA-RT methodologies are presented to analyze a mechatronic system

Researchers, Demri A. \& al. (2008) [6], proposed using SADT, FMECA (Failure Modes, Effects and Criticality 
Analysis), and the Petri nets methods to study the reliability of a mechatronic systems. By using functional analysis of defining the material limits, the various functions and operations performed by the system and the various configurations.

Researchers, Plateaux R. \& al. (2009) [7], proposed to integrate all of the part of the cycle $\mathrm{V}$ design in order to attain a continuity of the modeling through the different levels of approach and design (functional and structural requirements ). For this, they have proposed a hybrid method based on several tools, and Methods such as SADT, SysML (Systems Modeling Language) and Modelica, in the environment Dymola.

The researcher Vincent B. (2008) [8] applied the method Safe-SADT on a railway braking system on the one hand, and on the other hand he used this method to study the reliability of an automated system and to show the applicability of this method in industrial domains through a railway systems.

In order to study a production quality control system the researcher Khalaf Alahmad (2008) used the SADT method for constructing the Petri net model of a thermal central, and it evaluated the dynamic properties from the Petri network model. He studied the dynamic aspect of the system using the SADT temporal tool in the end; he produced a passage algorithm from SADT to Petri Network [9].

Works presented by Bruno Denis under the title "Design assistance and evaluation of the driving architecture of complex production systems" in (1994) [10] involve the design of the driving architecture of automated production systems". It's about a prospective work whose objective is to construct a formal methodological framework of design and architectures evaluation for using SA-RT, SADT and temporized Petri nets methods. To validate and clarify the proposed method he studied architecture design driving of a launch station with a free transfer. After presenting some research works on the application of some methodologies, a state of the art on the two methods SADT and SA-RT are presented.

\section{PRSENTATION OF THE MEthods SADT AND SA-RT}

The SADT represents an image of the system. It is a method of analysis to understanding why a system exists, or must be designed, what functions it must fulfill and finally, how they are realized, and whatever of the complexity. The method is based on a graphical model, proceeds by down approach in the sense that are going from general to more detailed, by focusing on system activity (Fig. 2) [11], [12].

The SADT method seems adapted to the modeling of mechatronic systems for at least one reason: this method applies perfectly to the multi-technological systems, that is to say, it adapts to mechanical, electronic and software systems [11], [13]. But it does not take into account the dynamic aspect of system.

The SART method is a method of specifying computer systems falling within of real time domains. It takes into account the dynamic aspect of the analyzed system. It is based on the SA method (Structured Analysis) which has been used extensively.

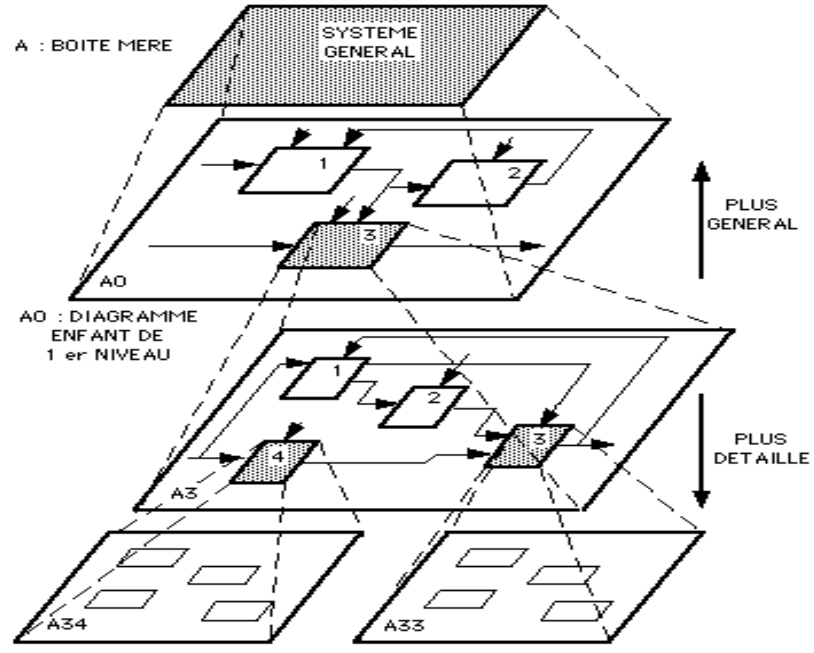

Fig. 2. Structure of an SADT model

SA-RT is well suited for applications with high dynamic behavior. This aspect is missing from the SADT method for modeling a mechatronic system [14].

In the SA-RT model, the definition of a system is given on the one hand of the description of system functions and control of the information flow and on the other hand of the description of the hardware architecture of the internal system (Fig. 3) [15], [16].

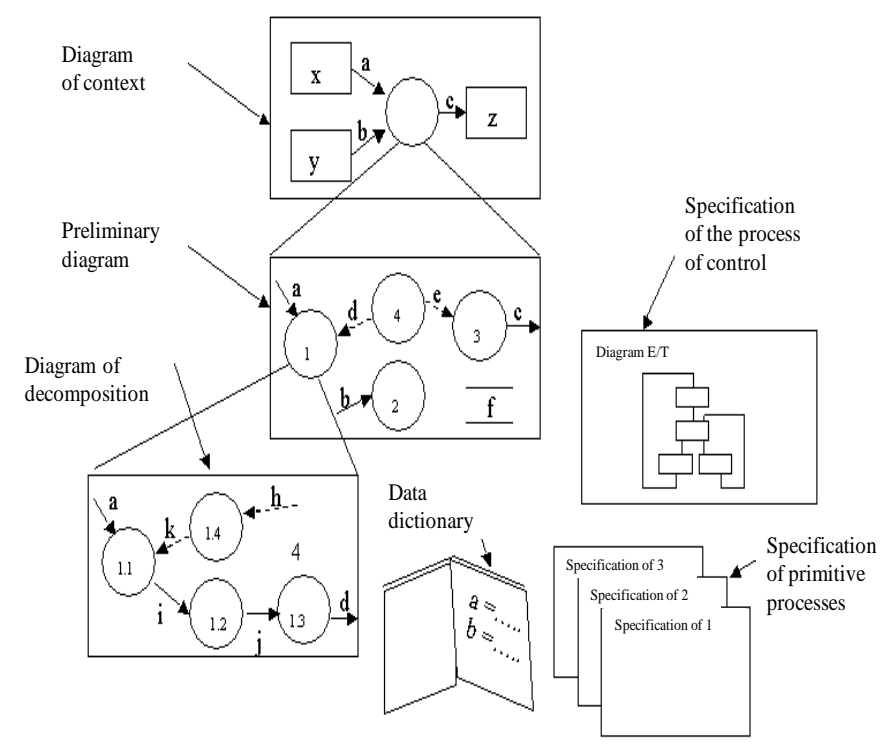

Fig. 3. Organization of the SA-RT model

\section{RESUlts OF THE ANALYSIS AND THE MODELING}

The presence of the ABS braking system in the vehicle is important, its functioning is based on an electronic calculator which constantly analyzes the speed of the vehicle and its variation, as well as of the four wheels (integrated sensors). When it detects a blockage of one or more wheels, the system responds by instructing the brake system and reducing its action on the designated wheel. Figure 4 shows the components of a braking system. 


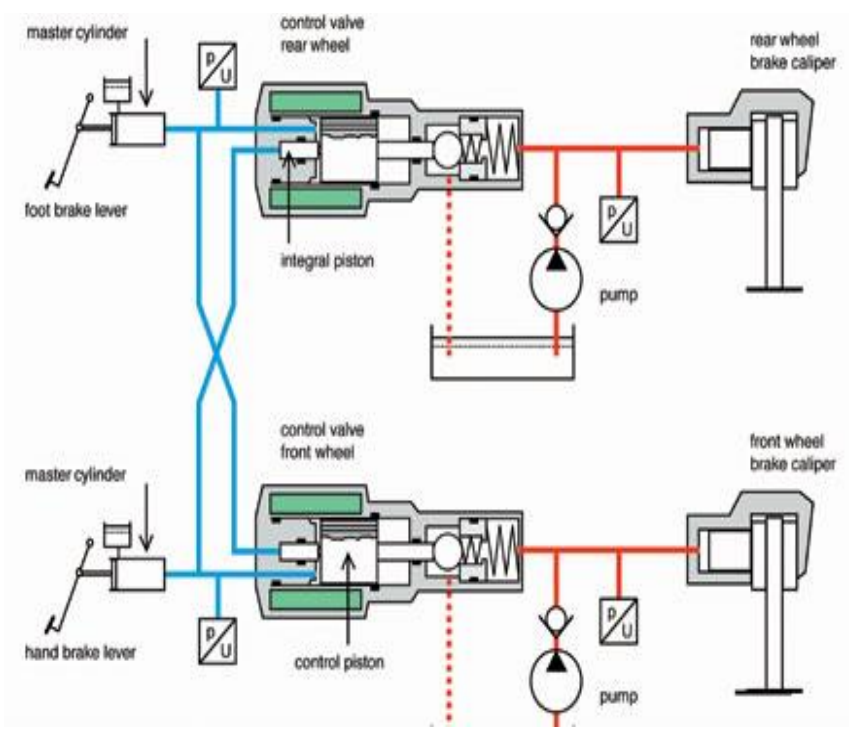

Fig. 4. Structure of an ABS braking system

\section{A. Review on braking system control}

Some studies on braking system control that has been presented in various researches:

Researchers Sidek S.N. \& al. (2000) [17], have considered the use of an intelligent controller to achieve the objective to modify the current conventional braking system so as to make it work automatically. To ensure high speed of system response, a DSP controller TMS320C24x with an embedded fuzzy algorithm is used in the implementation of this new device. Results of simulation studies using MATLAB have demonstrated the feasibility of this new system under investigation

Researchers Fletcher I. \& al. (2003) [18], have investigated one aspect of system design, the braking system. The design exercise is based upon a simulation of cars braking system enables several alternative control strategies to be assessed. The findings illustrate the problems involved and the opportunities available for the application of an 'intelligent' control strategy.

Researchers Li Junwei \& al. (2009) [19], have explained why $\mathrm{ABS}$ is an important part to improve the automobile's active safety. In general, ABS is designed to achieve maximum negative acceleration by preventing the wheels from locking. Researches show that the friction between road and tire is a nonlinear function of wheel slip. In this paper, to deal with the strong nonlinearity in the design of ABS controller, a variable structure controller has been designed and index reaching law and integral switching surface with saturation function methods are used to reduce chattering. In the simulations, several situations such as braking in dry road, wet road and snow road are considered.

Researchers Chun-Liang Lin \& al. (2011) [20], have presented how the slip ratio control problem in ABS is highly nonlinear and complicated. A sliding mode controller is developed to generate appropriate torque for the driving motor of two-wheel electric vehicles that ensures optimality of the slip ratio for efficient vehicle brake. The design is based on a novel short-circuit braking mechanism to emulate the mechanical ABS for the traditional gas-powered vehicles.

Researchers Lu Bo \& al. (2010) [21], have presented a simplified vehicle vertical two-wheeled model, an improved fuzzy PID controller was devoted to the ABS. This controller was obtained based on inosculating with fuzzy PID controller and the algorithm of objective function Automatic optimization. A simulation result was gotten through simulation of MATLAB Simulink environment. This result shows that the control system had better stability, adaptive, control precision and shortened the braking distance, braking time, reduced the slip angle through using this improved fuzzy PID controller. Comparing with the conventional PID controller, the fuzzy controller and the fuzzy PID controller, the better control effect and the control system stability could be acquired by this improved fuzzy PID controller.

Researchers Cabasino, M.P. \& al. (2011) [22], have considered the brake system of a vehicle whose wheels are equipped with ABS. They assume that the sensors that are responsible of the activation of the ABS are subject to faults. They first show how such a system can be modeled using labeled Petri nets and the notion of concurrent composition. Then, they show how fault diagnosis and diagnosability analysis can be performed on such a system using appropriate techniques based on Petri nets.

Researchers Qi Zhang \& al. (2004) [23], have presented the precondition of realizing logic thresholds based ABS which is the accuracy calculation of wheel angular acceleration. By analyzing the properties of the output signal of the electromagnetism induction sensor, which is used to measure wheel speed in vehicle antilock braking system, this paper proposes a kind of circuit that can satisfy the requirements of the system. Utilizing the wheel speed signals and applying the Kalman filter technology, the wheel angular acceleration is calculated. The trial results verify the feasibility and validity of this method.

Researchers Yonghua Xiong \& al. (2004) [23], have proposed an improved ABS ECU test system for the pneumatic ABS. Composed of finished ECU, data acquisition card, V/F convertor and $\mathrm{PC}$, the system accomplishes the establishing of vehicle, brake and braking air pressure model for realistic simulation, and the dynamic loading method is used to load the different model on PC. Using the system, the test and evaluation of the signal processing ability, communication capacity and the effectiveness of the controlling algorithm of ECU can be done. Finally, a type of ECU produced by one company is taken as an example to verify the feasibility of the testing system, and the results show that the system is available for practical application for its lowcost and short-cycle.

Researchers He Jidu \& al. (2004) [23], have presented a fuzzy immune adaptive PID control algorithm for ABS system after analyzed the lack of traditional slip rate ABS control algorithm, combining with the biological immune principle and the adaptive ability of fuzzy logic ratiocination. And the fuzzy immune PID controller adjust the proportional coefficient, the fuzzy controller adjust the integral and differential coefficient. Compared with the PID control algorithm, fuzzy control 
algorithm, fuzzy PID control algorithm and fuzzy immune adaptive PID control algorithm, the simulation results indicate that the method has characteristics of small overshoot, fast response, shorter braking distance and strong anti-interference ability and robustness. There is a higher application value in ABS control system.

\section{B. SADT results}

First, SADT analysis of the braking system ABS is presented. This analysis provides a synthetic description of the ABS system operating modes. The realization of this analysis is intended to identify the technical functions of the system. It allows us to make a hierarchical decomposition of the ABS system elements (Fig. 5).

The first diagram shown above is the level A-0 of the SADT method. The first diagram shows the main functions of the ABS system, which is to slow down the vehicle without blocking the wheels. The second diagram which is the level A0 shown in the following figure 6 is a decomposition of the previous level A-0, it contains 6 boxes.

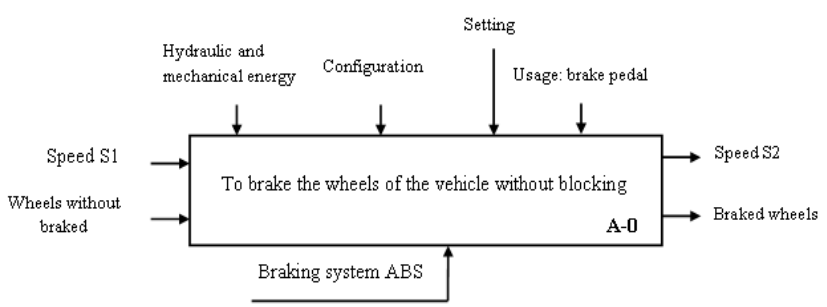

Fig. 5. Overall function of a braking system ABS

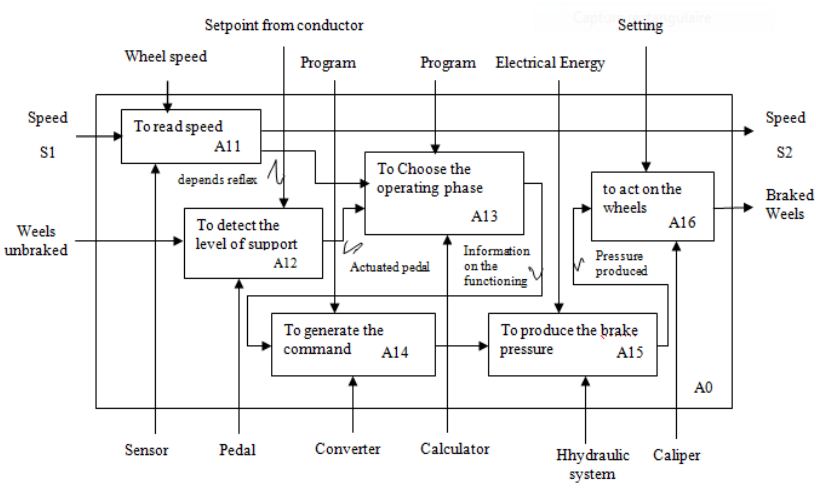

Fig. 6. Node A0 of the braking system

\section{SA-RT results}

Second, an SA-RT analysis of an automotive braking system composed on the one hand of a classic whole of a brake pedal (braking demand) and a brake (braking actuator) and on the other hand of an ABS system [24] is presented. A sensor sliding of wheel is associated to this ABS system. To simplify, the working of the ABS is based on a stop of braking when a sliding is detected on the wheels and it even though the driver's demand is always efficient. The driver has the possibility to activate this ABS system with the help of a specific button (button to two steady states: switch). A seer permits to indicate it (the activation of the ABS system). But then, it is not possible to deactivate the ABS system during the braking that is during the support on the brake pedal.

The whole of data or events exchanged with the outside of the functional process that represents the application, constitute specifications of entrances and exits of the application. The description of these Inputs/Outputs will be made in the dictionary of data.

The context diagram (Fig. 7) is constituted of the functional process "to control the braking system" and of five terminators:

- Brake pedal providing the data braking demand;

- Activation Button of the ABS providing the data ABS activation;

- Sliding sensor" providing the data Wheel sliding;

- Braking system consuming the data Braking command;

- ABS light consuming the data Display ABS.

This context diagram perfectly defines the interfacing between the inventor and the customer that is data to either provide or to generate [25].

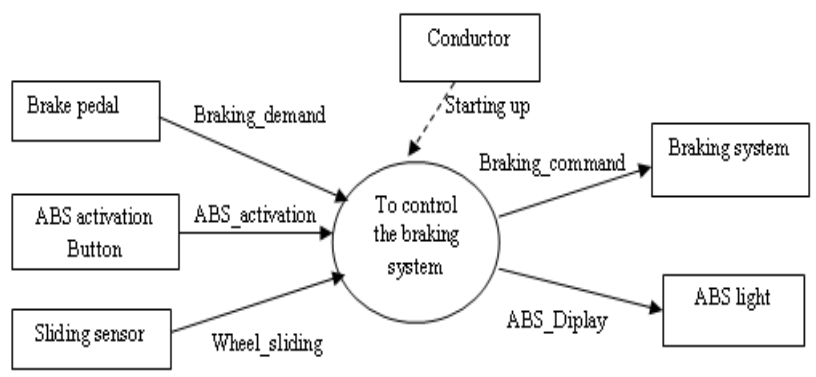

Fig. 7. Context Diagram of the braking system ABS

The preliminary diagram is constituted of five functional processes (Fig. 8). It can immediately underline at the level the obligatory consistency between the context diagram and the preliminary diagram at the level of the data flows in entrance and in exit. The passage of data between the functional processes is done of direct way. It is important to note that the data "Sliding_state" and the data "Button_ABS_state" are Boolean type.

A control process in the preliminary diagram is implemented in order to coordinate the different functional process execution (Fig. 9). This control process will therefore interact with a functional process either to launch or to activate its execution and, in return, the functional process will provide if necessary an event indicating the result of its treatment in order to give some useful information to change the control states. 


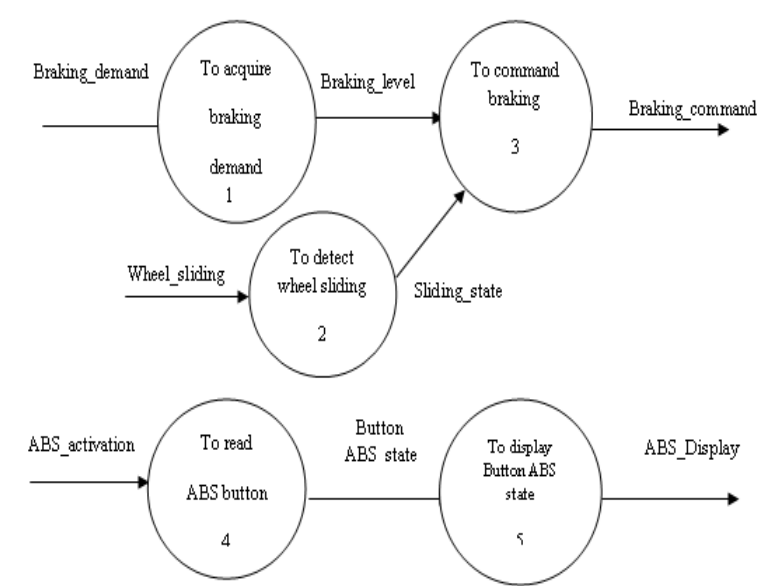

Fig. 8. Data Flow Diagram of the baking system ABS

In order to specify the control process of the application, the representation of the state-transition diagram is presented (Fig. 10).

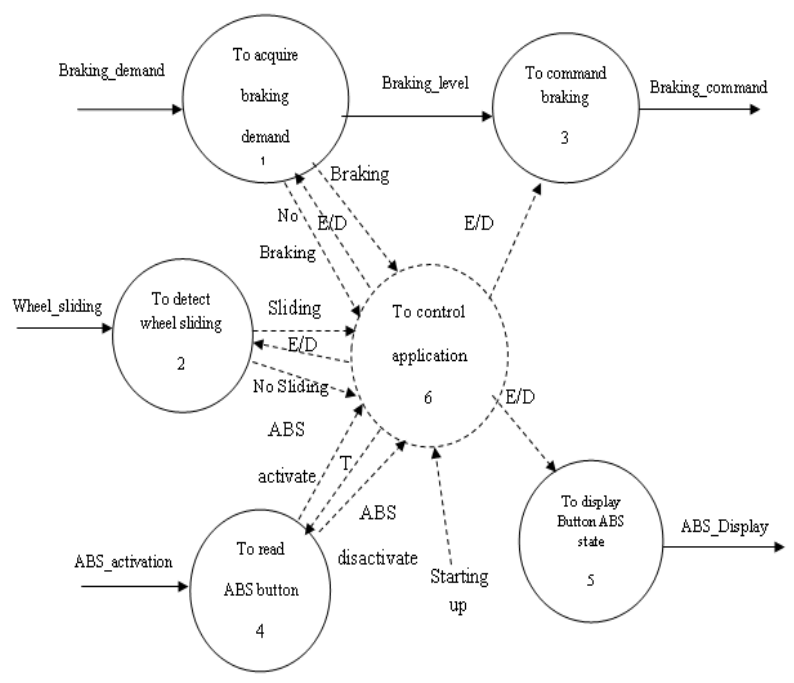

Fig. 9. Control Flow Diagram of the braking system ABS

The analysis of the system by the SADT method is descending, hierarchical, modular and structured, it brings out the maximum details as when in proportion as one progresses in the decomposition. The SADT representation system to describe the process in several levels to show the relationships, to explain the details gradually, controlled and summarized while avoiding the inaccuracies inherent in natural language. The SADT model allows clear view system functionality and interactions between these elements [37], [38].

After this analysis, we guarantee a functional description of the system regardless of the various possible solutions in the realization. Therefore it resulted in two levels of abstracti on, the conceptual level and an organizational level [9].

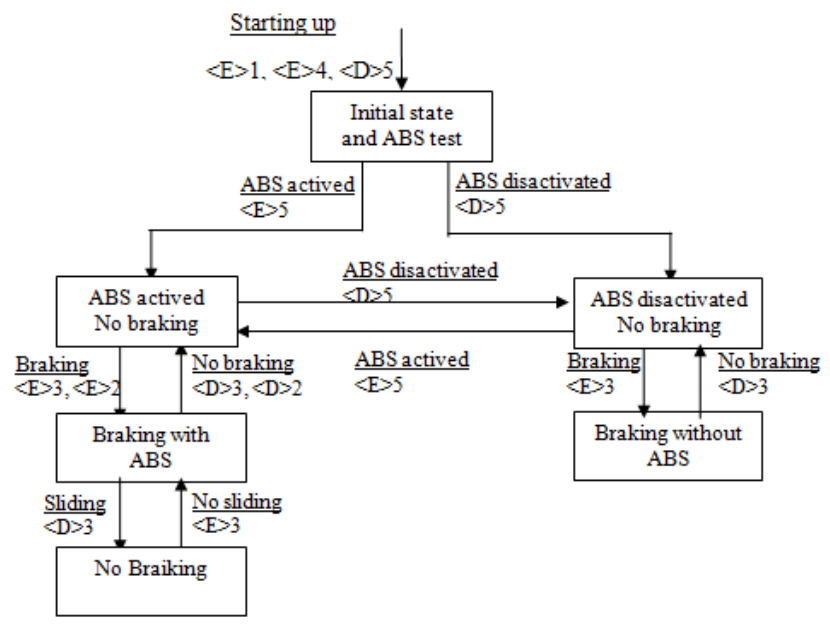

Fig. 10. State - Transition diagram of the ABS braking system

Watching the SADT model of the system it is noted that the depth of the decomposition depends on the informational availability between the levels. Furthermore, it is limited by the lower level of readability of the diagram. It contains a limited amount of information on a specific topic. Moreover it is difficult to analyze clearly the activities of a system with dynamic behavior [39], [40].

For the analysis of the system by the SA-RT method, it represents communication between designers and users of an application [30], [31], [32]. The SART models make it possible to express the wishes and needs of the user by removing the ambiguities of everyday language. SART is a move towards a method of dynamic specification. SART can thus be characterized by three aspects [33], [34], [35], [36]:

- A functional aspect, which is achieved through the use of data flow diagrams, help to show how functional processes transform their inputs (billows of incoming data) in outputs (billows of outgoing data). They also allow distinguishing between the types of discrete or continuous data, and they represent that the data storage can be used by multiple processes.

- An event aspect for the dynamic of the model is described by conditions or event occurrences. Two elements are introduced for this purpose: the control process, which pilots the functional processes by generation of events activation or deactivation [27] [28]; the control billows, representing the occurrence of events generated by different processes (functional or control). Here again, we can distinguish between consumables events (discrete) and permanent (continuous). The functioning of a control process is described via a state-transition diagram which models the life cycle processes. This diagram thus allows materialize the incidence of an event (or a combination of events) on the state of the system by indicating the actions to be taken when he arrived. 


\section{CONCLUSION}

In this paper, the need for system modeling of an ABS braking system for understanding its operation and exploitation with consistency and in a correct manner was presented. In fact, the methods used for the description of this system are characterized by their graph formalism which is necessary for the description of the system operating modes studied and identified sub systems and knowledge of its internal and external functions. Indeed, two structured analysis methods SADT and SA-RT are presented, on the one hand and an application of these methods on a practical case of an ABS braking system was presented, on the other hand. This application shows the interest of the graphical formalism of these two methods to the analysis and design of a mechatronic system in particular to describe the environmental and behavioral modeling.

Staring from this case study of the application of the structured analysis of an ABS braking system discussed in this paper, work is in progress to develop a general methodology of analysis and modeling for different mechatronic systems.

\section{REFERENCES}

[1] G. Turchany, Act together on Education for Sustainable Development, Bordeaux 27-290 October 2008.

[2] M.N. Lakhoua, Systemic analysis of an industrial system: case study of a grain silo, Arabian Journal for Science and Engineering, vol.38, 2013, pp. 1243-1254.

[3] El Feki M., Analysis and tolerance synthesis for the design and dimensioning of mechatronic systems, Central School of Lyon, 2011.

[4] Web site: http://www.engr.ncsu.edu/mechatronics/what-mech.php [consulted January 2016]

[5] M. Zerelli, Mechatronic systems with variable parameters: behavior analysis and tolerancing approach, LISMMA SUPMECA Toulon, École Centrale Paris, 2014.

[6] A.Demri, F. Charki, A. Guerin and H. Christofol, Functional and dysfunctional analysis of a mechatronic system, Annual Reliability and Maintainability Symposium RAMS, 2008, pp. 114-119.

[7] R. Plateaux, J.Y. Choley, O. Penas and A. Riviere, Towards an integrated mechatronic design process, IEEE International Conference on Mechatronics, ICM, 2009, pp. 1-6.

[8] B. Vincent, Application of the safe-sadt method on a railway braking system INRETS ESTAS, Villeneuve d'Ascq, France.

[9] K. Alahmad, Control system of production quality control methodology of modeling and optmization of production systems. Univ. Paul Verlaine in Metz, 2008.

[10] A. Bruno, Assistance in the design and evaluation of the architecture of conduct of complex production systems University Research Laboratory Automated Production (LURPA - EA 1385) Cachan, 1994.

[11] P. Jaulent, SADT a langage for communication, IGL Technology, Eyrolles, Paris, 1989.

[12] M. Galinier, SADT a language to communicate, Eyrolles, Paris 1989.

[13] Augusto, Modeling of complex systems. Higher National School of Mines of Saint-Etienne, 2013.

[14] Demri, Contribution to the evaluation of the reliability of a mechatronic system functional and dysfunctional modeling, Univ. Angers, 2009.

[15] D.J. Hatley, I.A. Pirbhai, Specification, Strategies for Real-Time Systems (SA-RT), Masson, Paris, France, 1991.

[16] M. N. Lakhoua, Using structured analysis for the control of real-time systems, Journal of Engineering and Technology Research Vol. 4(5), pp. 82-88, October 2012.

[17] S.N. Sidek and. M.J.E Salami, Design of intelligent braking system, Vol.2, 2000, pp. 580- 585.
[18] Fletcher I., Automatic braking system control, IEEE International Symposium on Intelligent Control, 2003, pp. 411- 414.

[19] L. Junwei and J. Wang, Design of antilock braking system based on variable structure control, IEEE International Conference on Intelligent Computing and Intelligent Systems, ICIS 2009.

[20] L. Chun-Liang and Y. Meng-Yao, Design of anti-lock braking system for electric vehicles via short-circuit braking, Second International Conference on Mechanic Automation and Control Engineering (MACE), 2011.

[21] Lu, Bo; Yu Wang; Jing-jing Wu; Jin-ping Li, ABS system design based on improved fuzzy PID control, Sixth International Conference on Natural Computation (ICNC), 2010

[22] M.P. Cabasino, A. Giua, C. Seatzu, A. Solinas and K.. Zedda, Fault diagnosis of an ABS system using Petri nets, IEEE Conference on Automation Science and Engineering (CASE), 2011.

[23] Qi Zhang; Guofu Liu; Yueke Wang; Tingting Zhou, Study of calculation method of wheel angular acceleration in ABS system, International Conference on Information Acquisition, 2004.

[24] Yonghua Xiong; Xiaoyan Li; Yong He; Min Wu; Yonghong Long, Design and application of testing system for pneumatic ABS ECU, 11th World Congress on Intelligent Control and Automation (WCICA), 2014.

[25] He Jidu; Zheng Yongjun; Tan Yu; Wu Gang, Research on Vehicle Anti-braking System Control Algorithm Based on Fuzzy Immune Adaptive PID Control, Third International Conference on Digital Manufacturing and Automation (ICDMA), 2012.

[26] F. Cottet, Real time systems of control command, Dunod., Paris. 2005.

[27] W. S. Liu, Real-time Systems, Prentice Hall, 2000.

[28] M.N. Lakhoua, Review of Structured Analysis and System Specification methods, Journal of Electrical Engineering, Vol.10, $\mathrm{N}^{\circ} 2$, 2010.

[29] D. Laurent, Contribution to the management of the regularity of execution of real-time tasks from one application to strict constraints, in an online scheduling context. Laboratory of Computer Science and Industrial Scienti_que (LISI), University of Poitiers, 2002.

[30] L.E. Colmenares-Guillen, O. Niño Prieto and A. Aguila Jurado, An Approach of Real-Time System for River Monitoring and FloodWarning System in Puebla, Mexico, World Applied Programming, Vol.3, Issue (8), August 2013. 328-340.

[31] M.I. Capel-Tuñón, Modelling and Simulation of a Real-Time Hybrid System, Proceedings of EOMAS'08.

[32] C. de la Riva and J. Tuya, Automatic generation of assumptions for modular verification of software specifications, Journal of Systems and Software, Vol.79, Issue 9, Sept 2006, pp. 1324-1340

[33] L. Jóźwiak and S-A Ong, Quality-driven model-based architecture synthesis for real-time embedded SoCs, Journal of Systems Architecture, Vol.54, Issues 3-4, March-April 2008, pp. 349-368.

[34] J.M. Fernandes, J. Lilius and Dragos Truscan, Integration of DFDs into a UML-based model-driven engineering approach, Softw Syst Model 2006, 5 pp. 403-428.

[35] M. Al-Mohamed and D. Esteve, Tools and models for systems design and synthesis of MEMS based on asynchronous circuits, IEEE International Conference on Industrial Technology vol.1, 2000, pp. 64 69.

[36] Specifying systems and applications with SA/SD/RT method, Training course: Modeling/SA, Insoft November 2013, web: http://www.insoft.fi

[37] D.A. Marca, SADT/IDEF0 for Augmenting UML, Agile and Usability Engineering Methods, Software and Data Technologies, 2012, pp. 3855.

[38] N. Ouasli, R. Ben Mehrez and L. El Amraoui, Parameter estimation of one wheel vehicle using nonlinear observer, International Conference on Electrical Sciences and Technologies in Maghreb, CISTEM 2014.

[39] M.N. Lakhoua, Contributions à l'analyse systémique, à la supervision et à la sûreté de fonctionnement des systèmes de contrôle-commande, Rapport de synthèse, HDR, ENICarthage, Tunisie, 2015.

[40] M.N. Lakhoua, Application of Functional Analysis on a SCADA system of a Thermal Power Plant, Advances in Electrical and Computer Engineering journal, 9(2), 2009. 